matter was not resolved! Opinions differed about the role of multivariate statistics, the criteria for establishing population diversity, the association of culture with any particular hominid group, the interpretation of bone 'tools' and the significance of differences in endocast morphology; in fact few 'stones' were left unturned.

Despite differences in approach, perhaps an uncomfortable level of broad agreement was reached about the existence of cranial, dental and postcranial morphotypes (though one suspects that for some participants there were too many cranial morphotypes to fit the teeth or postcranial material!). Future research problems that were identified include more comparative work on endocranial morphology, dental wear patterns and postcranial variability and further research into the implications of dental eruption patterns of hominines and current taphonomic studies for hominid behaviour.

This conference successfully brought together workers whose research approaches are different, but whose common aims are to discover more about the structure and behaviour of early man. This meeting complemented the workshop conference held in Nairobi in September 1973, which attempted to synthesise the results of work at Omo and East Rudolf. The publications resulting from these two meetings will attest to the growing scientific maturity of hominid studies and show the success of the international and multidisciplinary approach to hominid palaeontology which has been a feature of the research both at Omo and at East Rudolf.

\section{Are accretion disks stable?}

from a Correspondent

THE stability of accretion disks in binary X-ray sources comes under critical review in an article by Lightman and Eardley in a recent issue of Astrophysical Journal (187, L1; 1974). The accretion of material by a compact object (a neutron star or a black hole) from its companion in a binary star system is thought to be the main source of power for the luminous X-ray sources that have been discovered within our galaxy during the past few years. Because of the rotation of the binary system, transferred material is forced by the conservation of angular momentum initially to form a disk around the compact object. Differential rotation and viscosity, in the disk, transfer angular momentum outwards, allowing the matter to spiral inwards, liberating its gravitational potential energy as it does so. If the compact object is a neutron star, most of the energy is liberated at the surface of the star itself, where the accreting material crashes into it. But, if the compact object is a black hole, then all the luminosity comes from the disk, and the matter arriving at the inner edge of the disk is swallowed quietly by the black hole. Thus for accretion by a black hole, widely speculated to be the case for the X-ray source Cygnus $X-1$, the stability of the accretion disk is an all important matter.

The most uncertain element in all models of accretion disks is the viscosity -ordinary molecular viscosity is far too small, and authors usually invoke turbulence or magnetic fields. A common (ad hoc) assumption is that the effective kinematic viscosity (with dimensions of length ${ }^{2} /$ time) is roughly equal to the vertical thickness of the disk (the supposed size of a typical turbulent eddy) times some constant fraction of the sound speed (since supersonic turbulence would be rapidly dissipated). The difficulty arises in the inner regions of the disk where radiation pressure, rather than ordinary gas pressure, dominates the vertical structure. In this region, it is unclear what the 'sound speed' relevant to turbulent motion should be, and it is usually assumed to be that dictated by the radiation. Lightman and Eardley, however, have demonstrated that, in this part of the disk, this assumption about the viscosity is not consistent with the disk being in a steady configuration. They show, moreover, that if the disk is forced initially to conform to the previously calculated 'steady' configuration, it evolves secularly, breaking up into rings. The reality of these rings is, however, questionable, since their formation depends on being able to start in a supposed 'steady' situation, which has already been shown not to be self-consistent.

The reason for this 'instability' is fairly simple. The assumptions mentioned earlier lead to the conclusion that in this part of the disk, the viscous stress is inversely proportional to the surface density of the disk. Thus, if a small region of the disk has a lower than average surface density, the viscous stress, and hence the radial velocity, there will be higher than average, and so the surface density will decrease still further.

The authors suggest two possible alternatives. First, if one assumes rather that the 'sound speed' relevant to turbulent viscosity is that given by the gas pressure, then the resultant steady accretion is self-consistent. Second, if the assumptions made are roughly correct, then the idea of a steady accretion disk must be abandoned. The observed variability of the source Cygnus $\mathrm{X}-1$ on timescales as short as milliseconds might lead credence to this idea. In any event this contribution serves as a timely reminder to theoretical astronomers that 'ad hoc' assumptions which seem to work should not have too great a trust put in them.

\section{Darwin glass related to tektite fall?}

from our Geomagnetism Correspondent

The material that has come to be known as Darwin glass was first recognised and described just 60 years ago by Suess who found large quantities of it along a north-south track some $3 \mathrm{~km}$ to the east of Mount Darwin in Tasmania. About 50 years later, geochemical studies and the discovery that the glass contains coesite led to the view that the Darwin glass must have been formed by impact-a conclusion which was strikingly confirmed two years ago when Ford (Earth Planet. Sci. Lett., 16, 228 ; 1972) discovered Darwin Crater, a circular depression with a diameter of about $1 \mathrm{~km}$ lying some $4 \mathrm{~km}$ or so to the east of the most southerly of Suess's deposits.

It is now known that there are far more examples of glass in the vicinity of Mount Darwin than Suess had realised; and with the aim of confirming the genetic relationship between the original occurrences and the more recently discovered crater glasses, Gentner et al. (Earth Planet. Sci. Lett., 20, 204; 1973) have used both potassium-argon and fission track methods to date glass samples from the interior and rim of the crater and from areas up to $2 \mathrm{~km}$ away from the rim. The mean potassiumargon and fission track ages are $0.70 \pm$ 0.08 and $0.74 \pm 0.04$ million years, respectively, giving a combined age of $0.73 \pm 0.04$ Myr. This compares with the fission track age of $0.72 \pm 0.02$. Myr for the longer-known glasses, obtained previously by Gentner et al. (Geochim. cosmochim. Acta, 33, 1075; 1969).

Gentner and his colleagues thus conclude that all the known Darwin glasses were produced at the time of the impact that formed Darwin Crater 0.73 Myr ago. But there may be more in this than simply a study of the relations between glass deposits. That the Darwin glasses occur geographically close to the Australasian tektites has been obvious for some time; and what Gentner et al. have now done is to show that the glasses and the tektites have the same age. This is apparently the first evidence obtained that an impact crater was formed at the time of the Australasian tektites. It seems hardly likely that this is a coincidence; and yet it is unlikely that an impact forming a crater as small as the Darwin Crater could be 